\title{
Metodología para la deconstrucción dialógica de los estereotipos y roles de género en Valores Éticos.
}

Eduardo Elvira Jiménez ${ }^{a}$ y Esther Rivas Rivero ${ }^{b}$.

aUniversidad de Alcalá de Henares, colaborador UAH eduardoelviraj@hotmail.com , buniversidad Alcalá de Henares, Profesorado Asociada Área de Psicología del Desarrollo y de la Educación, Esther.rivas@uah.es.

\begin{abstract}
The scientific literature shows that gender roles and stereotypes are constructions related to social, cultural, religious, political factors, etcetera, that perpetuate sexist behaviours and attitudes through gender roles and stereotypes that discriminate against women and maintain inequality, legitimizing gender violence. The aim of the following study is to know these sexist and stereotyped conceptions of both women and men are developed and constructed using the dialogical deconstruction methodology. For this purpose, a qualitative approach design was developed with a sample of 38 adolescents from public institutes whose main technique was the Delphi Method, which was triangulated using participant observation and discussion groups. The results report that the students are more aware of equality, however, they maintain stereotyped conceptions about gender roles, being those referred to men the most prescriptive. The differences in the discourse of the adolescents seem to indicate that although progress has been made in social awareness about equality, it has no yet been internalized on the individual level.
\end{abstract}

Keywords: Dialogical learning, social desirability, gender stereotypes, gender roles, sexism, adolescence.

\footnotetext{
Resumen

La literatura científica demuestra que los roles y estereotipos de género son construcciones relacionados con factores sociales, culturales, religiosos, politicos, etcétera que perpetúan de las conductas y actitudes sexistas a través de los roles y estereotipos de género que discriminan a las mujeres y mantienen la desigualdad, legitimando la violencia de género. El objetivo del siguiente estudio es conocer cómo se desarrollan y construyen dichas concepciones sexistas y estereotipadas tanto de las mujeres como de los hombres haciendo uso de la metodología de la deconstrucción dialógica. Para ello se desarrolló un diseño de enfoque cualitativo con una muestra de 38 adolescentes de institutos públicos cuya técnica principal fue el Método Delphi, el cual se trianguló haciendo uso de la observación participante y los grupos de discusión. Los resultados parecen indicar que el alumnado se muestra más concienciado sobre la igualdad, sin embargo, mantienen
} 
concepciones estereotipadas sobre los roles de género, siendo aquellos referidos a los hombres los más prescriptivos. Las diferencias en el discurso de los y las adolescentes parecen indicar que si bien se ha avanzado en la concienciación social sobre la igualdad todavía no se ha interiorizado en el plano individual.

Palabras clave: Aprendizaje dialógico, deseabilidad social, estereotipos de género, roles de género, sexismo, adolescencia.

\section{Introducción}

La desigualdad y la discriminación ejercida contra las mujeres es todavía hoy una realidad tanto en contextos sociales y laborales como en la propia pareja. Los cambios tanto socioculturales como económicos, así como la incorporación de la mujer al mundo laboral, han mejorado su situación y ha permitido un mayor empoderamiento de las mujeres. Sin embargo, estos cambios no han sido suficientes para paliar la desigualdad que sufren las mujeres. De esta forma, la discriminación por el hecho de ser mujeres que antes sufrían a través del sexismo hostil ha dado paso al sexismo benevolente (Expósito, Moya y Glick, 1998), una forma de discriminación más velada y sutil (Lameiras y Rodríguez, 2002) pero que mantienen una concepción discriminatoria contra las mujeres atendiendo a su supuesta inferioridad.

El sexismo puede ser conceptualizado como toda aquella evaluación, actitud o conducta sobre una persona derivado de su sexo (Expósito et al., 1998). Allport (1954) consideraba el sexismo actitudes de antipatía hacia las mujeres, derivados de prejuicios hostiles construidos sobre ellas (Cameron, 1977). De esta forma, autores y autoras como Arnoso, Ibabe y Elgorriaga (2017) consideran el sexismo como aquellas creencias, actitudes o conductas conducentes a la discriminación y desigualdad contra las mujeres únicamente por el hecho de ser mujeres, sirviendo, a su vez, el sexismo como legitimación de la desigualdad y discriminación (Ovejero, Yubero, Larrañaga y Navarro, 2013). Dichos prejuicios derivan en conductas y actitudes de control y superioridad de los hombres sobre las mujeres ante su supuesta inferioridad (Alberdi y Rojas, 2015). Glick y Fiske (1996) indican la existencia de dos tipos de actitudes sexistas: por un lado el sexismo hostil son aquellas concepciones negativas y prejuicios sobre las mujeres basadas en la inferioridad de las mujeres y la creencia de que ciertas actitudes y conductas de las mujeres pueden ser peligrosas para el hombre al ser consideradas peligrosas y manipuladoras (Ferrer, 2017), lo cual conlleva a legitimar el control y el uso de la violencia contra las mujeres (Ovejero et al., 2013). Por otro lado, el sexismo benevolente mantiene concepciones negativas de la mujer respecto a su supuesta inferioridad referida a los hombres, sin embargo, en lugar de actitudes violentas para su control, este sexismo adquiere conductas de afecto y protección por parte de los hombres, legitimando de esta manera su control a través del paternalismo 
protector. Esto conlleva a mantener concepciones estereotipadas y restrictivas de las mujeres limitándolas a determinados roles (Castro, Fernández, Fernándes y Garrido, 2009), siendo sus cualidades complementarias a las de los hombres (Ferrer, 2017).

La desigualdad y discriminación contras las mujeres que el sexismo legitima se basa en los estereotipos y roles de género. Los estereotipos son aquellas preconcepciones que realizados de la realidad que observamos (Lippmann, 1922), o las creencias y concepciones sobre un determinado colectivo o grupo para ser capaces de entenderla de una forma simplificada (Morales y Huici, 2000). De la misma manera, Allport (1954, p. 191) considera los estereotipos como una creencia exagerada asociada a una categoría, cuya función es justificar o racionalizar nuestra conducta en relación con la categoría. Así, los estereotipos sirven para evaluar, analizar y comprender a determinados colectivos o grupos (Delgado-Álvarez, Sánchez y Fernández-Dávila, 2012; Poncela, 2014). Así, los estereotipos se determinan dependiendo de las creencias y expectativas que tengamos de un determinado grupo o colectivo (Gaviria, López Sáez y Cuadrado, 2013). Sirviendo como categorización social (Tajfel, 1969) y siendo transmitidos dentro del endogrupo a través de mecanismos socio-culturales (Del Valle, 2002).

Los estereotipos de género son aquellos estereotipos generados fruto de las creencias y expectativas socio-culturales que se tienen respecto a las conductas y actitudes tanto de hombres como de mujeres (Cuadrado, 2007). De esta manera, los estereotipos de género son las características prescriptivas que se asignas tanto a los hombres como a las mujeres atendiendo al sexo (Defago, 2015) siendo estos construidos y aceptados socialmente y estableciéndose como guías implícitas y prescriptivas de las conductas esperadas en cada sexo (Ferrer, 2017; Morales et al., 2007), siendo, por lo tanto, la base para las normas y pautas sociales que regulan las conductas de ambos sexos (Colás y Villaciervos, 2007). Siendo considerado negativo por parte de la sociedad la ruptura con los estereotipos de género, limitando y discriminándolas (Vázquez-Cupeiro, 2015).

Como indican López-Sáez y Morales (1995), los estereotipos de género se relacionan profundamente con los roles de género, siendo los primeros la base de los segundos, determinando de esta manera el rango de conductas y actividades esperadas atendiendo al sexo. Los roles de género pueden ser conceptualizados como las creencias consensuadas sobre las características de los varones y de las mujeres que se convierten en normativas para cada sexo sobre lo que debe o no hacer (Ferrer, 2017, p. 147). Es decir, los roles de género son aquellas expectativas relativas a hombres y mujeres atendiendo al género asociado a su sexo que indican aquellas conductas que se espera socialmente que realicen de acuerdo a los estereotipos de género (Aguilar, Valdez y González Arratia, 2013a). De esta manera, y de acuerdo con Baron y Byrne (2005), los roles de género son una construcción social que determina desde la forma de comportarse, de sentir y de pensar tanto de hombres como de mujeres de acuerdo a dos roles de género tradicionales definidos como masculinidad y femineidad. Si bien cada persona adquiere de forma individual los roles de género, estos son desarrollados e interiorizados a través de un proceso de aprendizaje de las funciones y concepciones que la sociedad tiene atendiendo a los estereotipos de género de forma social y en comunidad (Aguilar et al., 2013b; Barberá, 
1998), de esta manera los roles de género sirven para establecer relaciones de poder en desigualdad entre ambos sexos atendiendo a los estereotipos y roles de género (Vázquez, 2012). Se establece por tanto para los hombres roles de género relacionados con el sostenimiento económico de la familia, otorgando a las mujeres labores de cuidado de la familia y el hogar, adoptando un rol complementario e inferior respecto a los hombres, estableciendo a los hombres como los máximos responsables de su familia (Bravo, 2013; Lizana, 2012). Relacionando a los hombres con los ámbitos político-económicos y las mujeres con los ámbitos privado y familiar (Melero, 2010), lo cual se traduce en discriminación y desigualdad contra la mujer (López Méndez, 2007). Sin embargo, la naturaleza de construcción socio-cultural de los roles de género sin base en la biología o fisiología de los seres humanos (Gálvez, 2011), implica que no son estables e inmutables sino cambiantes y dependientes del contexto socio-histórico y cultural (Rubio, 2012).

\section{Objetivos.}

Le metodología seleccionada y utilizada es aquella relacionada con la deconstrucción dialógica. Dicha metodología busca la deconstrucción de los conceptos de género, estereotipos y roles de género, sexismo, mitos del amor romántico e influencia de los medios de comunicación y TIC en su reproducción y perpetuación para, finalmente, favorecer una reconstrucción crítica y reflexiva de significados a través del diálogo y el discurso con el alumnado y entre el alumnado. La deconstrucción dialógica se basa en el concepto de deconstrucción de Heidegger (Rodríguez García, 2012), la hermenéutica interpretativa de Gadamer (Gadamer, 1996), la acción comunicativa de Habermas (Merino, 2011; Noguera, 1996) y el aprendizaje dialógico de Freire (Freire, 1993).

El objeto de estudio son las concepciones que los y las adolescentes españoles tienen respecto a conceptos como el género, los estereotipos y roles de género, el sexismo, la violencia, la influencia de la cultura y la sociedad en su construcción, así como la importancia de los medios de comunicación y las TIC en su perpetuación y reproducción, tratando de favorecer la adquisición de conocimientos curriculares de la asignatura de Valores Éticos desarrollando un concepto de igualdad más natural y reflexivo a través de la deconstrucción dialógica. Partiendo de las siguientes hipótesis:

- El alumnado español muestra concepciones estereotipadas y prescriptivas respecto a hombres y mujeres atendiendo al género de acuerdo a los estereotipos y roles de género, debido esto a la influencia de la sociedad a través de los medios de comunicación y las TIC.

- El uso de la deconstrucción dialógica permite el desarrollo y la interiorización de un concepto de igualdad de forma reflexiva y crítica a través de un proceso de reflexión y deconstrucción de los conceptos previamente mencionados y su comprensión de su naturaleza como construcción socio-cultural, política y religiosa.

\subsection{Desarrollo de la innovación}

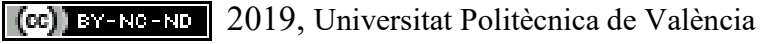


La muestra se seleccionó atendiendo a los principios de idoneidad, representatividad y accesibilidad dado que se realizó como parte de la asignatura de Valores éticos en las prácticas denominadas de investigación-acción (Elliott, 1990). De esta forma, la muestra consta de 38 participantes de segundo de la E.S.O. de un instituto público de Castilla La Mancha (39,5\% de alumnos y $60,5 \%$ de alumnas).

\subsubsection{Diseño y procedimiento.}

La metodología desarrollada basa su referencial teórico en el enfoque cualitativo y la estrategia utilizada es concurrente de triangulación. La técnica principal de recogida de información y transformación social utilizada fue el Método Delphi (Gil, 2011) adaptado a la investigación cualitativa. De esta manera se desarrolló una serie de cuestionarios de respuesta abierta ad hoc que se entregó online al alumnado para su cumplimentación de forma reflexiva y crítica anónimamente. Una vez respondidos por el alumnado fueron leídos y tanto las preguntas como las respuestas reformuladas y devuelvas con el correspondiente feedback, las cuales debían ser respondidas de nuevo por el alumnado en sucesivas rondas hasta alcanzar el punto de saturación de la información recogida y de la deconstrucción realizada. Para la credibilidad de los datos se recurrió a la triangulación, para ello se utilizó la observación participante (Pérez Juste, Galán y Quintanal, 2012) tanto durante las sesiones de Valores Éticos como en los recreos. Además, se realizaron grupos de discusión o focales dado sus potencialidades a la hora de recoger información sobre temas sensibles atendiendo no sólo a la información verbal sino también a las no verbal y las actitudes (de Lara y Ballesteros, 2007; Gil, 2011; León y Montero, 2015), dichos grupos de discusión o focales se realizaron en dos fases: en la primera el criterio de agrupación fue el sexo para permitir la expresión sin la presión grupal; en la segunda fase se realizaron los grupos de discusión o focales con ambos sexos para comparar tanto las respuestas como las actitudes.

\subsection{Resultados.}

El alumnado de la muestra reporta que existen diferencias en la concepción de hombres y mujeres referidas al género. De esta manera, mientras parte del alumnado considera que no se dan diferencias entre los hombres y las mujeres, otra parte del alumnado sí los considera de forma diversa, tanto biológicamente como de acuerdo a su estereotipo de género. Esto conlleva a que parte del alumnado conciba como una misma dimensión tanto el género como el sexo, no siendo capaces de concebir su diferenciación.

Destaca cómo algunas alumnas de la muestra no consideran de forma estereotipada a las mujeres pero sí mantienen el estereotipo tradicional de masculinidad referido a los hombres, esperando de ellos conductas y actitudes consideradas masculinas como puede ser que sean fuertes, duros, heterosexuales, etcétera.

La mayoría del alumnado de la muestra se relaciona de acuerdo al género asociado a su sexo, sin embargo, no consideran que esto deba ser prescriptivo, indicando que se puede dar 
la situación de que un hombre no se identifique con su género ni una mujer con el suyo. Consideran que puede ser la sociedad quienes influyen y determinen en nuestras conductas, actitudes, pensamientos y sentimientos referidos al género y a los estereotipos y roles de género.

Si bien el alumnado sí considera diferente a hombres y mujeres atendiendo al género además de a los aspectos biológicos y fisiológicos, no conciben que existan actividades masculinas o actividades femeninas ni que unas actividades sea prescriptivas de un sexo $\mathrm{u}$ otro. La explicación que llegaron a dar respecto a la concepción social de las actividades masculinas o femeninas hace referencia al aprendizaje y la educación recibida de generación en generación derivado de la evolución de las sociedades hacia la diferenciación de sexos. De forma que el alumnado indicó cómo aquellas personas que se distancian de su estereotipo y rol de género tienden a ser rechazados por la sociedad.

Ante las cuestiones relacionadas con la perpetuación y mantenimiento de los estereotipos y roles de género, el alumnado expresó su creencia en la influencia de la transmisión de generación en generación, manteniéndose y perpetuándose actualmente a través de la influencia de los medios de comunicación y las TIC. Tanto medios de comunicación como TIC, son considerados por el alumnado como espacios donde se muestran los patrones y modelos de hombre y mujer que la sociedad acepta y los individuos deben aspirar a representar y desear, sirviendo como reguladoras de las conductas deseadas e inhibidoras de las conductas no deseadas. De esta manera, las mujeres y hombres mostrados en las redes sociales y medios de comunicación tienden a ser representaciones idealizadas del estereotipo masculino y femenino, relacionándolo a través de la sexualidad y el deseo sexual.

No obstante, el alumnado considera que las redes sociales ofrecen también un espacio donde mostrar nuevos modelos de hombres y mujeres alejados del estereotipo de masculinidad y femineidad. Siendo de esta manera herramientas para derribar los estereotipos y roles de género. Consideran en ello con especial importancia a los influencers y su influencia en las personas, especialmente adolescentes. De esta manera las redes sociales adquieren una doble vertiente como reguladoras de conductas y perpetuadoras de los estereotipos y roles de género, y como forma de expresar la propia identidad y combatir los estereotipos y roles de género a través de mostrar modelos alternativos a los estereotipos tradicionales.

Sin embargo, en la deconstrucción dialógica referida a los mitos del amor romántico no se consiguió el proceso de deconstrucción y reinterpretación esperado. De esta manera, el alumnado continuaba considerando los celos en la pareja como algo normal, siendo incluso concebido como algo natural al amor. Explicando los celos en la pareja como un proceso normal de preocupación por el bienestar y la pérdida de la persona amada. Una pequeña parte de la muestra consideraba los celos como algo negativo independientemente de la situación o contexto en que se den, aunque entendían el sentir celos consideraban que no se debe considerar normal ni perpetuar. Respecto a la consideración de los celos y sus implicaciones, el alumnado era capaz de considerar los celos como posesión, sin embargo, ésta era concebida de forma positiva haciendo referencia a que se trataba de una muestra de 
amor y preocupación. Sin embargo, una minoría en la deconstrucción dialógica llegó a considerar los celos como una construcción socio-cultural.

Para garantizar la credibilidad de los datos obtenidos en la clase de Valores Éticos obtenidos mediante la deconstrucción dialógica, se realizó una triangulación con grupos de discusión o focales en las clases de Valores Éticos y observación participante tanto en el aula como en otros contextos del centro escolar. Durante los debates cuyo criterio de agrupación fue el sexo, tanto chicos como chicas se expresaron abiertamente, sin embargo, en los debates en gran grupo los chicos se mostraban ajenos al debate, distantes y poco interesados en participar, tratando de no participar, algo que contrasta con el interés mostrado por las chicas del grupo, quienes en todo momento lideraron el debate con muchas intervenciones y sacando nuevos temas transversales al propuesto. Al pedir a algún chico en concreto su participación y que diera su opinión al respecto, estos trataban de evadir la respuesta y no ser preguntados. Al preguntar el motivo de su apatía y desinterés en el debate de gran grupo, nuevamente preferían no responder o respondían con evasivas. Al ser consultado el desinterés a las chicas del grupo, éstas mencionaron que no participan al ser considerado por ello como un tema de chicas y no mostrar interés en la igualdad entre sexos al no encontrarse ellos en una situación de desigualdad y discriminación. La diferencia de participación de los chicos referido al Método Delphi y en los grupos de discusión cuyo criterio de agrupamiento fue el sexo respecto al grupo de discusión en gran grupo hace pensar en la influencia del sesgo de deseabilidad social.

\subsection{Conclusiones}

Las dificultades encontradas en el alumnado de esta edad para concebir la diferencia entre sexo y género deriva dificulta el procesamiento adecuado de determinados aspectos relacionales y sociales derivados de la creencia de que el sexo y el género son una misma dimensión, provocando sesgos que pueden derivar en una mayor tendencia a los heurísticos desde la perspectiva de los estereotipos y roles de género, dificultando la concienciación e interiorización de la igualdad. Esto se observa especialmente en cómo la interiorización de los estereotipos de género, especialmente hacia los hombres, genera dinámicas de desigualdad y discriminación en la relación con el otro sexo al esperar unas determinadas actitudes y conductas. Por ello, y debido a la deseabilidad social, el alumnado de la muestra respuesta estereotipadamente, generando un inestable equilibrio entre los discursos feministas que reproducen y las concepciones sexistas que prevalecen en el alumnado. Los procesos de deconstrucción dialógica parecen haber posibilitado una concepción más crítica y reflexiva del alumnado en aspectos como el género, los estereotipos y roles de género, y el sexismo. Especialmente el alumnado rechaza el sexismo hostil, mostrándose más tolerante con las actitudes y conductas derivadas del sexismo benevolente $\mathrm{y}$, dependiendo del contexto y situación, legitimando el sexismo ambivalente, especialmente en aquellos aspectos relacionados con los mitos del amor romántico.

Las redes sociales y los medios de comunicación son identificados como una herramienta para la perpetuación y reproducción de los estereotipos y roles de género. Sin embargo, el 
uso que de ellas hacen las personas puede ser considerado como una forma de sensibilidad y concienciar sobre identidades y modelos alternativos a los tradicionales, como pueden ser las nuevas masculinidades.

La deseabilidad social ha sido un factor importante y muy presente en el estudio. De esta manera, se han observado respuestas del alumnado que se mostraba "transgresor" o concienciado y activista frente a los convencionalismos sociales y los estereotipos y roles tradicionales a través de la reproducción de los principales discursos feministas de actualidad. Sin embargo, en dichos discursos se observan incoherencias en aspectos menos tratados por el feminismo como los estereotipos y roles de género referidos a los hombres o algunas concepciones respecto a los mitos del amor romántico. Asimismo, se observan incoherencias entre lo respondido en los grupos de discusión y en el Método Delphi con lo observado en la observación participante. De esta manera el alumnado varón puede haber modificado sus respuestas y actitudes, especialmente en los grupos de discusión en presencia de sus compañeras, de acuerdo a aquello socialmente aceptado por temor a ser considerados machistas. De la misma manera, aunque la recogida de datos en el Método Delphi era anónimo, las alumnas de la muestra mostraron mucho interés en que supiéramos cuál era su número, de cara a que los investigadores pudiéramos saber cuáles eran sus respuestas y mostrarlas con orgullo y obtener nuestra aprobación. Lo cual indica que tanto las respuestas de tanto chicos como chicas pueden no ser creíbles en la fase exploratoria y estar influidas por la deseabilidad social.

Como limitaciones se podrían destacar la falta de colaboración y participación inicial del alumnado, especialmente del alumnado masculino de la muestra, lo cual dificultó el proceso de deconstrucción dialógica y reinterpretación de los conceptos. Obligando a separar los grupos de discusión en un primer momento atendiendo al sexo para permitir la libre y abierta expresión sin temor a decir algo que pudiera ser considerado sexista por parte de sus compañeras. Otro aspecto a destacar hace referencia al uso de una muestra no probabilística, incidental y reducida, seleccionando al alumnado previamente agrupado en la asignatura de Valores Éticos durante las horas de docencia. Finalmente, la deseabilidad social y la presión de grupo llevan a considerar que las respuestas pueden no haber sido sinceras en la fase exploratoria. En líneas futuras de investigación sería interesante realizar procesos de deconstrucción dialógica con alumnado universitario para comparar el proceso de construcción de significados e interpretaciones atendiendo a los diferentes momentos evolutivos. Otro aspecto interesante a explorar sería adaptar la metodología de deconstrucción dialógica para la adquisición de competencias y conocimientos de forma crítica y reflexiva, especialmente en campos de la educación superior como pueden ser la Educación y las Ciencias Sociales que permitan un enfoque más crítico y reflexivo y no meramente academicista, pudiendo comprender cómo la influencia de determinados Grados Universitarios influye en la construcción de significados y la reinterpretación de aquellos realizados durante su adolescencia.

En síntesis, la investigación pone de relieve la posibilidad de que no exista una conciencia crítica y reflexiva respecto a la igualdad y los estereotipos y roles de género. Siendo posible que el alumnado se limite a reproducir los discursos feministas socialmente aceptados 
actualmente con el objetivo de ser aceptados y aceptadas y no ser considerados sexistas, haciendo suyo un discurso que en ocasiones no comparten o no comprenden. La aparición de deseabilidad social puede dar lugar a dos interpretaciones: por un lado, el alumnado puede no considerar como algo negativo el sexismo, los estereotipos y roles de género, indicando lo contrario al ser lo socialmente aceptado; por otro lado, puede significar que se ha avanzado en la concienciación y sensibilización social, generando una conciencia social más igualitaria, si bien ésta no ha sido asimilada e interiorizada por el alumnado en el plano individual y personal todavía.

\section{Referencias}

Aguilar Montes de Oca, Y. P., Valdez Medina, J. L., González-Arratia López-Fuentes, N. I., y González Escobar, S. (2013b). Los roles de género de los hombres y las mujeres en el México contemporáneo. $\begin{array}{lllll}\text { Enseñanza e } & \text { envestigación } & \text { 207-224. }\end{array}$ $<$ https://www.redalyc.org/pdf/292/29228336001.pdf> [Consulta: 20 de marzo de 2019]

Aguilar Montes de Oca, Y. P., Valdez Medina, J. L., y González Arratia López Fuentes, N. I. (2013a). Satisfacción con los roles de género. Revista electrónica de psicología Iztacala, 15(4). $<$ http://campus.iztacala.unam.mx/carreras/psicologia/psiclin/vol15num4/Vol15No4Art14.pdf> [Consulta: 20 de marzo de 2019]

Allport, G.W. (1954). The Nature of prejudice. Cambridge, MA: Addison-Wesley.

Arnoso, A., Ibabe, I., Arnoso, M., y Elgorriaga, E. (2017). El sexismo como predictor de la violencia de pareja en un contexto multicultural. Anuario de Psicología Jurídica, 27(1), 9-20.

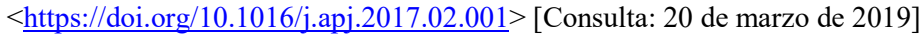

Barberá, E. (1998). Psicología del género. I. M. Benlloch (Ed.). Barcelona: Ariel.

Baron, R. A. y Byrne, D. (2005). Psicología Social. (10ª Ed.) Madrid: Pearson Educación.

Bravo, C. D. (2013). ¿Diferentes? Estudio de identidad y roles de género. Revista Sexología y Sociedad, 4(12), 36-39.

Cameron, C. (1977). Sex-role attitudes. En S. Oskamp (Ed.), Attitudes and opinions. Prentice Hall. 339359.

Castro, J. M. C., Lisboa, S. L. S. L., González, C., Carvajal, C. C., y Alegría, I. (2016). Inventario de sexismo ambivalente: adaptación, validación y relación con variables psicosociales. Salud \& Sociedad, 1(2), 125-135.<https://www.redalyc.org/pdf/4397/439742463006.pdf $>$ [Consulta: 20 de marzo de 2019]

Colás P., Villaciervos P. (2007) La interiorización de los estereotipos de género en jóvenes y adolescentes. Revista de Investigación Educativa, 25(1), 35-38.

Cuadrado, I. (2007). Estereotipos de género. En J. F. Morales, E, Gaviria, M. C. Moya e I. Cuadrado (eds.). Psicología Social (pp. 243-266). Madrid: McGraw-Hill.

De Lara Guijarro, E. y Ballesteros Velázquez, B. (2007). Métodos de investigación en educación social. Madrid: UNED.

Defago, M. A. P. (2015). Estereotipos de género: la perpetuación del poder sexista en los tribunales argentinos. $\quad$ Estudos $\quad$ Feministas, 23(1), 35-51. $<$ https://www.jstor.org/stable/pdf/estufemi.23.1.35.pdf?seq=1\#page_scan_tab_contents $>$ [Consulta: 20 de marzo de 2019]

Del Valle, T. (Coord.) (2002). Modelos emergentes en los sistemas y relaciones de género. Narcea. Madrid

Elliott, J. (1990). La investigación-acción en educación. Ediciones Morata.

Expósito, F., Moya, M. C., y Glick, P. (1998). Sexismo ambivalente: medición y correlatos. Revista de Psicología Social, 13(2), 159-169. <https://doi.org/10.1174/021347498760350641> [Consulta: 20 de marzo de 2019]

Ferrer Pérez, V. A. (2017) Feminismo y Psicología Social. Madrid: Grupo 5. 
Freire, P. (1993). Pedagogía de la esperanza: un reencuentro con la pedagogía del oprimido. Siglo xxi.

Gadamer, H. G. (1996/1964). Estética y Hermenéutica. Daimon Revista Internacional de Filosofia, (12), 512. <https://revistas.um.es/daimon/article/view/8311> [Consulta: 20 de marzo de 2019]

Gálvez, C. (2011). Violencia de género: Terrorismo en casa. España: Formación Alcalá.

Gaviria Stewart, E., López Sáez, M. Y Cuadrado Guirado, I. (2013). Introducción a la Psicología Social (2a Ed.) Madrid: Sanz y Torres.

Gil Pascual, J. A. (2011). Técnicas e instrumentos para la recogida de información. Madrid: UNED

Glick, P. y Fiske, S. T. (1996). The Ambivalent Sexism Inventory: Differentiating Hostile and Benevolent Sexism. Journal of Personality and Social Psychology, 70 (3), 491-512.

Lameiras, M. y Rodríguez, Y. (2002). Evaluación del sexismo moderno en adolescentes. Revista de Psicología Social, 17(2), 119-127 <https://doi.org/10.1174/021347402320007555> [Consulta: 20 de marzo de 2019]

León, O. G. y Montero, I. (2015). Métodos de investigación en psicologías y educación: Las tradiciones cuantitativa y cualitativa. Madrid: McGraw-Hill.

Lippmann, W. (1922). Public Opinion. Londres: Allen and Unwin.

Lizana, R. (2012) A mí también me duele: niños y niñas víctimas de la violencia de género en la pareja. Barcelona: Gedisa

López Méndez, I. (2007). El enfoque de género en la intervención social. España: Cruz Roja española.

López-Sáez, M., y Morales, J. F. (1995). Gender stereotyping in the Spanish Population: looking into the future. Gender, management and science, 151-168.

Melero, N. (2010). Reivindicar la igualdad de mujeres y hombres en la sociedad: una aproximación al concepto de género. Barataria: Revista Castellano-Manchega de Ciencias Sociales, (11), 73-83. $<$ http://dx.doi.org/10.20932/barataria.v0i11.152> [Consulta: 20 de marzo de 2019]

Merino, E. S. V. (2011). "Racionalidad, diálogo y acción: Habermas y la pedagogía crítica”. Revista Iberoamericana de Educación, 56(3), 1-15. <https://rieoei.org/RIE/article/view/1507> [Consulta: 20 de marzo de 2019]

Morales, F. y Huici, C. (2000). Psicología Social. Madrid: UNED.

Noguera, J. A. (1996) La teoría crítica: de Frankfurt a Habermas Una “traducción” de la teoría de la acción $\begin{array}{llllll}\text { comunicativa } & \text { a } & \text { la } & \text { sociología. } & \text { Papers } & \text { 133-153. }\end{array}$ $<$ https://www.raco.cat/index.php/papers/article/view/25424> [Consulta: 20 de marzo de 2019]

Ovejero, A., Yubero, S., Larrañaga, E., y Navarro, R. (2013). Sexismo y comportamiento de acoso escolar en adolescentes. Psicología Conductual, 21(1), 157.

Pérez Juste, R., Galán González A. y Quintanal Díaz, J. (2012). Métodos y diseños en investigación en educación. Madrid: UNED.

Poncela, A. M. F. (2014). Prejuicios y estereotipos. Refranes, chistes y acertijos, reproductores y transgresores. Antropología Experimental, (11). $<$ http://revista.ujaen.es/huesped/rae/articulos2011/22fernandez11.pdf $>$ [Consulta: 20 de marzo de 2019]

Rodríguez García, M. (2012). La construcción de la identidad desde su deconstrucción originaria: Heidegger y el humanismo. In Libro de Actas del I Congreso Internacional de Comunicación y Género. Sevilla, 5, 6 y 7 de Marzo de 2012. Dir. Juan Carlos Suárez Villegas; comité organizador Irene Liberia Vayá y Belén Zurbano Berenguer (pp. 290-305). Sevilla: Facultad de Comunicación. Universidad de Sevilla.

Rubio, S. P. (2012). Familia transnacional y redefinición de los roles de género. El caso de la migración boliviana en España. Papers: revista de sociologia, 97(3), 661-684. < https://www.raco.cat/index.php/Papers/article/view/255855> [Consulta: 20 de marzo de 2019]

Tajfel, H. (1969). Cognitive aspects of prejudice. Journal of Biosocial Science, 1(S1), 173-191. $<$ https://doi.org/10.1017/S0021932000023336> [Consulta: 20 de marzo de 2019]

Vázquez, J.J. (2012). Estereotipos de género. En E. Carmona (Ed.). Diversidad de género e igualdad de derechos. Manual para una asignatura interdisciplinar, 15-27. Valencia: Tirant Lo Blanch. 
Eduardo Elvira Jiménez y Esther Rivas Rivero.

Vázquez-Cupeiro, S. (2015). Ciencia, estereotipos y género: una revisión de los marcos explicativos. Convergencia, 22(68), 177-202. <http://www.scielo.org.mx/scielo.php?pid=S140514352015000200177\&script=sci_arttext\&tlng=en $>$ [Consulta: 20 de marzo de 2019] 